\title{
Asymmetric Synchronous Collaboration Within Distributed Teams
}

\author{
M. Ashdown and M. L. Cummings \\ Humans and Automation Laboratory, Massachusetts Institute of Technology, \\ 77 Massachusetts Avenue, Cambridge, MA 02139, USA, \\ http://web.mit.edu/aeroastro/www/labs/halab/
}

\begin{abstract}
Teams performing physical tasks must often be distributed in space, and are often organized hierarchically. This means that systems to support collaboration between members must account for the asymmetry in physical environment, organizational roles, and available technology. Using urban search and rescue as an example, we first describe the factors that cause this asymmetry. We then discuss the way information should be shared, and the type of awareness that should be supported. We suggest the use of very different display and interaction devices for operators at the organizational levels, to complement their situations and needs.
\end{abstract}

Keywords: synchronous remote collaboration, computer-supported cooperative work, command and control.

\section{Introduction}

A team of people with a task is often organized in a hierarchy, to allow it to scale with the magnitude of the task. This is especially true for command and control organizations in the military, and emergency response in the civilian sector. Team members must often be distributed geographically, so they communicate via radios, and more recently, with digital communications.

In this paper we consider situations in which a real-world task is performed, our example being urban search and rescue (USAR). The goal will be to search damaged buildings and extract victims. The search and rescue will be conducted by a combination of humans and robots with operators in the field, and these will be linked via a digital network to personnel at a base who supervise and plan the operation (Figure 1). The physical domain is dynamic so the personnel must react to it under time-pressure. We assume a network is available and that the team has access to technology that will be available in the near future. We address the problem of how technology should be designed to support synchronous collaboration between personnel in the field and those at the base.

Personnel in the field will be in a different environment, using different equipment, and performing different tasks from those at the base. This means that the information required by the the two classes of collaborators will be very different, as will the technology that can best support them. 


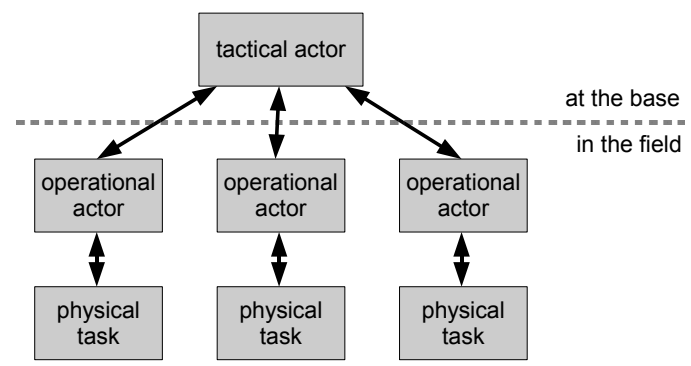

Fig. 1. We consider situations where a tactical actor is situated in a base and is supervising operational actors in the field. In the case of USAR, an operational actor's physical task may be searching and rescuing directly, or controlling a robot.

In this paper, we will describe the nature of the asymmetry inherent in hierarchies used to manage personnel in the field, highlight some issues for design of remote collaboration for this type of task, and suggest the combination of different display types and interfaces for creating a distributed synchronous collaboration system that supports the information needs and awareness of different classes of personnel.

\section{Hierarchical Organization}

Levels in a command hierarchy are typically labelled operational, tactical, and strategic: operational units perform their duties in the field, the tactical level allows multiple operational units to be supervised, and the strategic level makes long-term decisions governing the mission.

We are considering hierarchical organizations with distributed personnel that collaborate synchronously using voice and data channels, to operate in a dynamic domain. Examples include military command and control organizations, and the Incident Command System [3] used in crisis response. Other relevant domains include air traffic controllers and pilots, where Data Link communications have reduced time spent and distance flown [26], and dispatch of vehicles such as taxis, which have benefited from the use of GPS and digital networking [16].

The agents in such a system have conventionally been human actors, but these are now joined at the operational level by uninhabited vehicles (UVs), including robots, which must often be accompanied in the field by their operators. Conventionally, communication between locations has been achieved by speaking on radios, but synchronous digital exchange is now becoming available. Even in domains like crisis response where infrastructure may have been damaged, mesh networks can be formed by combining multiple portable devices and mobile network nodes positioned around an incident site. Sensing systems such as the global positioning system (GPS) can provide real-time data about the current situation.

Operational personnel will be working under time pressure, possibly in noisy, hazardous environments with limited ability to carry or use large, complex com- 
puting devices. In USAR, the primary task will be to search and rescue, or operate robots to search for victims using video cameras and other sensors. Communicating with the tactical level is an operator's secondary task, so it should not impede the primary one, and the communication device should be compact, unobtrusive, and simple to operate. Tactical personnel must deal with large amounts of information to form awareness of the whole situation, and then filter and fuse it before communicating it to the operational personnel. Thus, support should be provided for tactical actors to view a large amount of information simultaneously, but coherently.

Most remote synchronous collaboration technologies, such as videoconferencing and groupware [10], assume that the situations and roles of the collaborators are fairly symmetric, and that the data they share is not subject to external influences. In contrast, as discussed below, we are considering a class of domains where significant asymmetry exists between collaborators, and where the situation is dynamic: it progresses independently of the collaborators and it causes time-pressure. Advances in displays, networks, and sensing will make remote collaboration involving a multitude of real-time data possible in these domains. The design of collaboration systems should be driven by the fundamental physical, organizational, and technological asymmetries.

\section{Role Asymmetry}

There are major differences between the situations of the operational and tactical actors, and even between operational actors, performing a manual task is different from controlling a UV (Table 1). UV control falls between the other two conditions in many respects.

Table 1. Differences between operational and tactical situations

\begin{tabular}{|c|c|c|c|}
\hline & \multicolumn{2}{|c|}{ operational } & \multirow{2}{*}{ tactical } \\
\hline & manual & UV & \\
\hline $\begin{array}{l}\text { role } \\
\text { environment } \\
\text { latency } \\
\text { knowledge of world } \\
\text { knowledge of plans } \\
\text { computing }\end{array}$ & $\begin{array}{c}\text { execute actual task } \\
\text { difficult, hazardous } \\
<1 \text { second } \\
\text { local, detailed } \\
\text { narrow } \\
\text { portable, limited }\end{array}$ & $\begin{array}{l}\text { control robot to do task } \\
\text { noisy, distracting } \\
\text { seconds } \\
\text { local, depends on sensors } \\
\text { narrow, but plans av ailable } \\
\text { portable }\end{array}$ & $\begin{array}{c}\text { supervise operations } \\
\text { controlled } \\
\text { minutes } \\
\text { global, coarse } \\
\text { comprehensive } \\
\text { fixed, powerful }\end{array}$ \\
\hline
\end{tabular}

Role. The operational actor is situated in the field where the physical task occurs. This may be performed directly, or in our USAR example may involve the use of a robot. In contrast, the tactical actor's job is to supervise: she deals with monitoring, planning, and commanding.

Environment. The environment at the operational level presents various constraints because it is determined by external factors, may be restrictive in various ways, and may well require the operational actor to move from location to location. The environment in the base of operations for the tactical actor can be chosen to fit the needs of the task: we will assume it is fixed for the 
duration of the mission, is large enough to contain all the desired technology, and is protected from weather and other hazards.

Latency. Because the operational actor interacts directly with the external world, feedback will be fast, and the classical observe-orient-decide-act loop [9] will occur rapidly. For UV control, communication latency will affect this loop, thus degrading the human's interaction with the world. The tactical actor will work on longer timescales, and it may take some time to receive feedback on the effectiveness of a plan being executed. It may be possible for the tactical actor to take control of a UV from afar, but the end-to-end latency and reliabilty of the network will limit this.

Knowledge of world. An operational actor will possess detailed knowledge of the physical situation around him. The tactical actor will form a wider view that does not contain such fine detail, but does contain significantly more information from a wider variety of sources. A UV operator's view will depend on the sensors available: for instance, proprioceptive sensors that sense which direction is up, and whether a robot's wheels are turning, will help to diagnose problems. Information must be aggregated as it is passed up from operational to tactical to avoid information overload, and similarly the tactical actor needs to select which information is useful before contacting any particular operational actor.

Knowledge of plans. The tactical actor is charged with forming and maintaining a plan for the whole team, so she will have knowledge of the whole plan. An individual operational actor needs to know the details, only for the part corresponding to her own task. The tactical level should transmit updates to the plan to the operational level as needed. Compared to a manual operational actor, because a UV operator's primary task is performed with a computer, she should have more opportunity to stay aware of changes to the plan displayed on her screen.

Computing. The physical constraints at the operational level mean that any computing device must be small and portable, so the power of the device and the amount of information it can display will be limited compared to the tactical level. The tactical actor may be in a permanent command centre, or a mobile base of operations, but in either case state of the art computing and display facilities should be available.

Velez et al. [27] found that in remote collaboration, role asymmetry combined with platform heterogeneity impacts collaboration. For instance, they found that in a two-person system, when one collaborator has a restricted view of a task due to hardware constraints, some status is conferred onto the other, who will have a tendency to take charge. Technological constraints, such as asymmetric processing power and network bandwidth, will affect the design of collaboration systems [13]. However, other fundamental constraints should be addressed, such as differences in attention available from the user, differences in display devices, and noisy environments at the operational level. 


\section{Remote Collaboration}

We are considering situations where the tactical actor collaborates remotely with the operational actors he is supervising. Advances in networking will allow the medium for collaboration to consist of data and graphics, in addition to the conventional voice channel. Below we describe some issues in designing additional collaboration channels, and we emphasize the importance of supporting awareness, both at the operational and the tactical level.

The case of UV tasking provides an extra possibility over conventional command hierarchies, because tactical personnel may be given the ability to take control of some or all of the capabilities of the UVs at the operational level, subject to network limitations. Also, operations involving heterogeneous systems may involve the tactical operator supervising some UVs directly while collaborating with operational personnel controlling other UVs in the field.

\subsection{Sharing Information}

The standard audio channel used in many command and control situations may now be augmented with a digital channel. Using cameras for collaboration will not be very useful in this case; instead, a digital channel can be used to share visual information that represents task status, acting, and planning. This can be called a task space, as opposed to a person space [4]. It allows the collaborators to transmit information more precisely than when speaking, ground their conversations on the information they share, and perform visual gestures that may be much simpler than their verbal equivalents.

A geographic information system (GIS) displaying maps and GPS locations is a prime example of the type of large-scale dynamic data that can be displayed at the tactical level to aid in situation assessment and planning. In our USAR example, a dynamic map might contain buildings to be searched, the locations of operational personnel and robots, and additional information useful for the planning activities. The tactical actor will want to view the whole map. During synchronous collaboration with an operational actor, a portion of the map may be shared between the two to allow deictic references; collaborators can point and annotate as they talk over the voice channel about plans and actions.

Mulgand et al. [17] consider data shared at three distinct levels: pixels, data, or recipes. Sharing pixels creates a what you see is what I see system, provides common ground in the conversation, but restricts the collaborators to having identical views. Sharing data allows collaborators to see customized views of the same data. Sharing recipes is the most flexible because each collaborator can modify the recipe - this is essentially the database query used to generate the data. However, this means collaborators may end up viewing quite different

representations of the same situation, so they will have to rely more heavily on the voice channel to resolve discrepancies.

\subsection{Awareness}

It is important to support awareness in a collaborative system. A dynamic domain will require awareness of both the changing situation in the external world, 
and of the data and plans that the collaborators share. Gutwin and Greenberg [11] define four basic characteristics of awareness:

1. Awareness is knowledge about the state of a particular environment.

2. Environments change over time, so awareness must be kept up to date.

3. People maintain their awareness by interacting with the environment.

4. Awareness is usually a secondary goal - that is, the overall goal is not simply to maintain awareness but to complete some task in the environment.

Workspace awareness [11] means knowing what other collaborators are doing in a shared workspace, and can be supported by views of the actions of others such as radar views, which show a miniature version of a whole workspace so that the actions of others are visible. The deictic references mentioned in the section above are a form of intentional communication, whereby a collaborator explicitly signals to another, but consequential communication, whereby one collaborator becomes aware of the actions of another by watching them happening, is also important. This happens automatically during collocated collaboration, but in a distributed system, features to support this type of communication must be explicitly added. One example is feedthrough: for example, a graphical object can give visual feedback of the operations performed on it, for the benefit of remote observers. This could be used if one collaborator was adding points of interest to a map, to allow another to watch them being created. In addition, network reliability and latency will effect the design of the shared task space. For example, higher latency will require more persistent remote gestures because it will be harder for collaborators to synchronize with each other.

In a dynamic domain it is not just workspace awareness regarding shared digital data that is necessary, but also situation awareness [7]: knowing what is going on in the external world. This consists of perceiving the elements of the world, comprehending what they mean, and predicting future states. Generally it is a challenge to get information passed up from the operational to the tactical level, to allow perception of the situation at the higher level. Advances in portable computational devices and sensing technologies will allow much data on the state of operations to be automatically transmitted in real-time up to the tactical level. However, because the tactical actor is working at a higher level of abstraction, it will probably be necessary to distil the low-level data into a status visualization that can be rapidly assessed. In particular, the many sensors fitted to UVs will allow large amounts of information to be sent up to the tactical level, but visualizations should be devised that avoid cognitively overloading tactical personnel.

It is important to note that collaborators must remain aware, not only of raw data and the external situation, but also of the plans of the agents in the system, and how well they are fulfilling them. This could be called activity awareness [5], and presumes a method for actors to express their plans to the computer.

\section{Technology}

Ubiquitous computing as envisaged by Weiser [29] occurs in an environment populated by computing devices of different sizes-tabs, pads, and boards. Stan- 
dard computer screens are pad-sized, at 15-21 inches. The tab-sized, 2-4 inch displays of mobile phones and personal digital assistants have become popular, and recently there has been significant research interest in the board-sized displays, of 60 -inches or more, that can now be constructed. The quantitative size differences between these devices entail qualitative differences in the activities they may support.

We propose supporting asymmetric collaboration between actors in a distributed, organizational hierarchy by combining very different computing devices. At the operational level, personnel will use use small displays on portable devices, due to the constraints of the physical situation and because their organizational role requires that they spend most of their time on their physical task rather than manipulating data. At the tactical level, personnel will use large displays that allow a significant amount of data to be monitored and manipulated. The data will consist of information about the current situation, such as maps of a search site with locations of the units, and future plans, such as lists of buildings to search and victims to extract and treat.

\subsection{Portable Devices}

Advances in areas such as batteries, wireless networks, and small screen technology are fuelling an upsurge in the use of portable computing devices, but Raghunath et al. [18] argue that "advances in technology will not significantly mitigate handhelds' limitations because human perceptual and motor systemsnot the underlying technology - are the real limiting factors." These factors include human visual acuity, which limits the amount of information that can be displayed on a handheld, and size, which makes it impractical for multiple people to view or interact with such a small device.

The use of handheld computers in the field that allow real-time communication between remote actors has been pioneered by pervasive games [2]. Handhelds have also been used successfully in medical settings [25]. There has been some work on combining patients' screens, which are primarily used for entertainment, with such handhelds [1].

\subsection{Large Displays}

In the controlled environment of a base of operations, tactical actors will be free to exploit the benefits of the large display devices that are becoming available. Rather than the low-resolution shared wall displays that are often used in command centres, we mean large high-resolution personal displays. The Office of the Future [19] pioneered the use of large displays in an office environment. Infrastructure for large displays has received considerable attention [8], and includes multi-projector calibration, and systems for visualization of large datasets. LambaVision [15] is an architecture for combining an array of flat-panel monitors into a single large display; currently the seams between monitors are visible, but advances in hardware may soon allow them to disappear. Interfaces for such displays have recently received much attention, particularly for collocated collaboration [21], and recent developments in multi-touch sensing [12] hold the promise of more fluid interfaces. 
A large display is qualitatively different from a small one, and large displays have been shown to have benefits for cognition [23] and productivity [6]. Swaminathan \& Sato [22] cite the ability to collaborate with several other people, and the ability to present a primary work object in context as a important benefits of such displays. They state that a large contiguous display, such as an interactive desk, is most useful for applications with the following characteristics:

1. A large amount of interrelated information needs to be displayed, and

2. any part of this information could, at any time, become the centre of user's attention and may need to be carefully studied or modified.

In our USAR task, such a device would allow the simultaneous display of detailed maps, plans for the operation, and status of operational units. All this information must be considered at the tactical level, but can be condensed into a simple form for each operational unit, such as a prioritized list of buildings to search.

Large displays have different affordances as compared to conventional ones, and pose different challenges. For instance, a mouse pointer can easily get lost, window management requires different mechanisms, and conventional menus are awkward to use when the menu bar is far away. A large display, even if technically homogenous, will not be used in a homogenous manner by the user, and the much larger space makes it possible to simultaneously display much more information, which enables more complex multitasking behaviour [20]. The realization that the standard Window Icon Menu Pointer (WIMP) interface does not scale to very large displays has led to a series of modifications to the standard interface, and also to some radical re-inventions, including multi-touch finger and pen input, gesture recognition, arbitrarily rotated and scaled items placed in 'bins', user interface widgets that respond differently to each user, and physical objects used to control the digital content $[14,21]$.

\subsection{Collaboration Between Screen Sizes}

Screen size affects performance in tasks, for instance the lack of context when looking up items in a large table on a small screen reduces performance [28]. Tremaine et al. [24] had remote users collaborate synchronously on a puzzle task using either a conventional personal computer or a handheld computer, with the handheld offering a more restricted view of the problem. The results in the PC-handheld and PC-PC conditions were better than those in the handheldhandheld condition, because if at least one person had a good view of the task by using the PC, they could help the other person. In that case the person on the PC had the necessary situation awareness, which was remotely shared.

When using a shared view of a map, the tactical actor with the large display may be given control of the viewing parameters because she has the context necessary to make informed decisions about where to look. In general, the tactical actor may naturally assume much of the control of the shared data because this is the domain in which she works, while the operational actor is primarily dealing with his physical task. Thus the use of different display devices will complement the physical environments and organizational roles of the collaborators. 


\section{Conclusion}

In a hierarchical crisis management organization, it is desirable to have tactical actors situated in a secure base, but operational actors must often be located in the field. Advances in displays, networks, and sensing will allow synchronous remote collaboration between these two levels. However, organizational asymmetry, and cognitive and physical constraints at the operational level, will mean that technology for collaboration should reflect the differing roles and situations of the collaborators.

A task space between distributed collaborators can be used to share information that is difficult to convey verbally. The level of sharing - pixels, data, or recipes - must be chosen. Also, support should be provided for the workspace, situation, and activity awareness of all actors in the system. We have proposed the use of display devices of very different sizes at the different organizational levels. At the operational level, small, simple, portable devices will support the viewing of individual plans and indication of status information. At the tactical level, a large display will allow for the comprehension of the complete situation and formulation of a plan that includes multiple operational units.

In our future work we will implement a USAR system for synchronous remote collaboration using different display devices to complement the work of actors in a hierarchical organization, as described above. We will design interfaces to support the types of awareness listed above, and perform experiments to validate our design and provide recommendations for similar systems.

Acknowledgements We thank Gilles Coppin and Stacey Scott for their valuable comments and insights. This research was conducted in conjunction with Thales Research and Technology (UK) and funded by European Commission FP6 Marie Curie Outgoing International Fellowship number 21743.

\section{References}

1. O. A. Alsos and D. Svanaes. Interaction Techniques for Using Handhelds and PCs Together in a Clinical Setting. In Proc. NordCHI, pages 125-134, 2006.

2. S. Benford, C. Magerkurth, and P. Ljungstrand. Bridging the Physical and Digital in Pervasive Gaming. Communications of the ACM, 48(3):54-57, 2005.

3. G. A. Bigley and K. H. Roberts. The Incident Command System: High-Reliability Organizing for Complex and Volatile Task Environments. Academy of Management Journal, 44(6):1281-1299, 2001.

4. W. Buxton. Telepresence: Integrating Shared Task and Person Spaces. In Proceedings of Graphics Interface '92, pages 123-129, 1992.

5. J. M. Carroll, D. C. Neale, P. L. Isenhour, M. B. Rosson, and D. S. McCrickard. Notification and awareness: synchronizing task-oriented collaborative activity. Int. J. Human-Computer Studies, 58(5):605-632, 2003.

6. M. Czerwinski, G. Smith, T. Regan, B. Meyers, G. Robertson, and G. Starkweather. Toward Characterizing the Productivity Benefits of Very Large Displays. In Proceedings of IFIP Interact 2003, pages 9-16, 2003.

7. M. R. Endsley. Toward a Theory of Situation Awareness in Dynamic Systems. Human Factors, 37(1), 1995.

8. T. Funkhouser and K. Li. Large-Format Displays (introduction to special edition on large displays). In IEEE Comp. Graph. \& $A$ App., pages 20-21, 2000. 
9. T. Grant and B. Kooter. Comparing OODA \& Other Models as Operational View C2 Architecture. In Proc. 10th ICCRTS, 2005.

10. J. Grudin and S. E. Poltrock. Computer-Supported Cooperative Work and Groupware. Advances in Computers, 45:269-320, 1997.

11. C. Gutwin and S. Greenberg. E. Salas, S. M. Fiore and J. A. Cannon-Bowers (Eds) Team Cognition: Process and Performance at the Inter-and Intra-individual Level, chapter The Importance of Awareness for Team Cognition in Distributed Collaboration. APA Press, 2004.

12. J. Y. Han. Low-Cost Multi-Touch Sensing through Frustrated Total Internal Reflection. In Proceedings of UIST 2005, pages 115-118, 2005.

13. A. M. Krebs, B. Dorohonceanu, and I. Marsic. Supporting Collaboration in Heterogeneous Environments. J. Management Inf. Sys., 20(4):199-227, 2004.

14. G. Kurtenbach and G. Fitzmaurice. Applications of Large Displays (introduction to special edition). IEEE Comp. Graph. \& App., 25(4):22-23, 2005.

15. J. Leigh et al. The Global Lambda Visualization Facility: An International UltraHigh-Definition Wide-Area Visualization Collaboratory. In Future Generation Computer Systems, pages 964-971, 2006.

16. Z. Liao. Taxi Dispatch vis Global Positioning Systems. IEEE Transactions on Engineering Management, 48(3):342-347, 2001.

17. S. Mulgand, A. Travis, J. Standard, C. Means, and A. Burgman. Shared User Interfaces for Dynamic Collaboration in Network-Centric Command Centers. In Proc. ICCRTS, 2005.

18. M. Raghunath, C. Narayanaswami, and C. Pinhanez. Fostering a Symbiotic Handheld Environment. In IEEE Computer, pages 56-65, 2003.

19. R. Raskar, G. Welch, M. Cutts, M. Lake, L. Stesin, and H. Fuchs. The Office of the Future: A Unified Approach to Image-Based Modelling and Spatially Immersive Displays. In Proceedings of ACM SIGGRAPH 98, pages 179-188, 1998.

20. G. Robertson, M. Czerwinski, P. Baudisch, B. Myers, D. Robbins, G. Smith, and D. Tan. The Large-Display Experience. IEEE Comp. Graph. \& App., 25(4):44-51, 2005.

21. S. Scott and S. Carpendale. Interacting with Digital Tabletops (introduction to special edition). IEEE Comp. Graph. \& App., 26(5):24-27, 2006.

22. K. Swaminathan and S. Sato. Interaction Design for Large Displays. Interactions, 4(1):15-24, 1997.

23. D. S. Tan, D. Gergle, P. G. Scupelli, and R. Pausch. With Similar Visual Angles, Large Displays Improve Spatial Performance. In Proc. of CHI 2003, pages 217-224, 2003.

24. M. Tremaine, A. Sarcevic, D. Wu, M. C. Velez, B. Dorohonceanu, A. Krebs, and I. Marsic. Size Does Matter in Computer Collaboration: Heterogeneous Platform Effects on Human-Human Interaction. In Proc. 38th Annual Hawaii Int. Conf. System Sciences (HICSS 2005), page 33, 2005.

25. P. Turner, G. Milne, M. Kubitscheck, I. Penman, and S. Turner. Implementing a Wireless Network of PDAs in a Hospital Setting. Per. Ubiq. Comp., 9(4):209-217, 2005.

26. User Benefits of Two-Way Data Link ATC Communications. Technical Report DOT/FAA/CT-95/4, US Department of Transportation, 1995.

27. M. Velez, M. Tremaine, A. Sarcevic, B. Dorohonceanu, A. Krebs, and I. Marsic. "Who's in Charge Here?" Communicating Across Unequal Computer Platforms. ACM Trans. CHI, 11(4):407-444, 2004.

28. C. Watters, J. Duffy, and K. Duffy. Using Large Tables on Small Display Devices. Int. J. Human-Computer Studies, 58(1):21-37, 2003.

29. M. Weiser. The Computer for the 21st Century. Scientific American, 1991(Sept):94-104, 1991. 